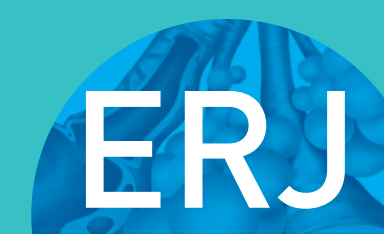

open research
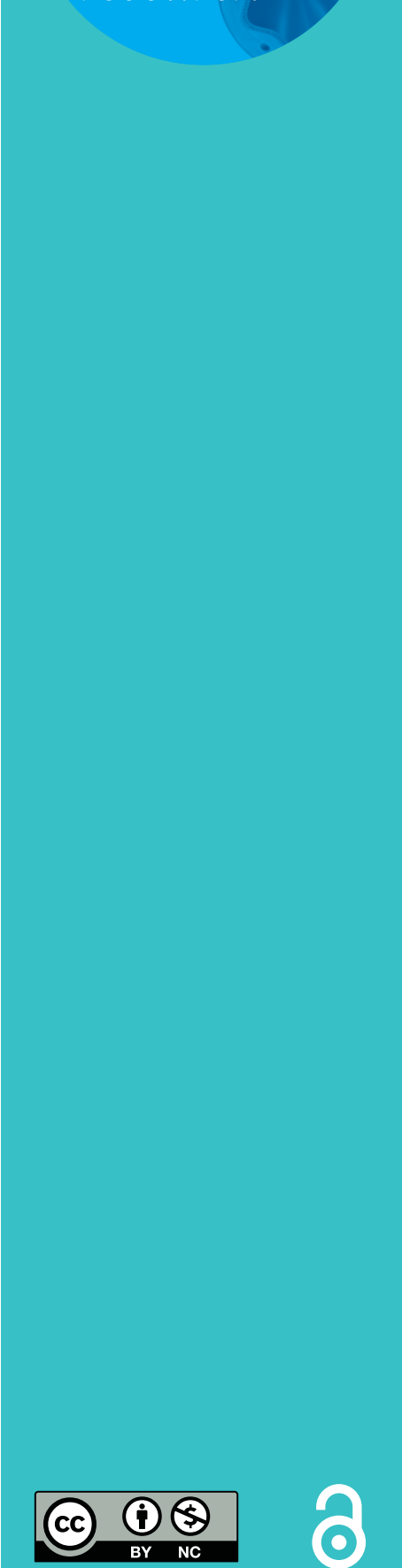

\section{The economics of precision health: preventing air pollution-induced exacerbation in asthma}

\author{
Tima Mohammadi ${ }^{1}$, Mohsen Sadatsafavi (10 ${ }^{2}$ and Chris Carlsten $\mathbb{1}^{3}$
}

Affiliations: ${ }^{1}$ Centre for Health Evaluation and Outcome Sciences, University of British Columbia, Vancouver, BC, Canada. ${ }^{2}$ Collaboration for Outcomes Research and Evaluation, Faculty of Pharmaceutical Sciences, University of British Columbia, Vancouver, BC, Canada. ${ }^{3}$ Air Pollution Exposure Laboratory, Division of Respiratory Medicine, Dept of Medicine, University of British Columbia, Vancouver, BC, Canada.

Correspondence: Chris Carlsten, 2775 Laurel Street, 7th Floor - The Lung Centre, Vancouver, BC, V5Z 1M9, Canada. E-mail: carlstendamail.ubc.ca

ABSTRACT The demonstrable value of precision medicine, in the context of common environmental exposures, has scarcely been explored. This study evaluated the cost effectiveness of a preventive personalised intervention to reduce the adverse effect of air pollution in the context of asthma.

A decision-analytic model was used to conduct a cost-utility analysis of prevention interventions in case of acute exposure to air pollution in mild asthma. Three different strategies, as follows, were compared: no preventive intervention; precision health strategy based on information from genotype testing, followed with treating high-risk patients; and prescribing additional medication to all mild asthmatics as a preventive intervention. The costs and quality-adjusted life years (QALYs) in the base case and alternative scenarios were obtained through probabilistic analysis.

The results showed that the precision prevention intervention (anticipatory intervention for asthmatics, guided by relevant genetic abnormality, in the face of acute air pollution) is a cost-effective strategy compared with no such intervention, with an incremental cost-effectiveness ratio of CAD 49555 per QALY. Furthermore, this strategy is a dominant strategy compared with an intervention that prescribes medication indiscriminately to all asthmatics.

The incorporation of genomic testing to stratify risk of asthmatics to pollution-driven exacerbations, and then tailoring a preventive intervention accordingly, may be cost effective relative to untailored methods. These results lend plausibility to the use of precision medicine for limiting asthma exacerbation in the context of air pollution and, potentially, other exposures.

@ERSpublications

Glutathione- $S$-transferase genotyping to determine the use of preventive asthma medication in the face of air pollution is cost effective in this model. Precision prevention in the setting of common environmental exposures may be used in other contexts. https://bit.ly/35Lab4b

Cite this article as: Mohammadi T, Sadatsafavi M, Carlsten CThe economics of precision health: preventing air pollution-induced exacerbation in asthma. ERJ Open Res 2021; 7: 00790-2020 [https:// doi.org/10.1183/23120541.00790-2020].

This article has supplementary material available from openres.ersjournals.com

Received: 27 Oct 2020 | Accepted: 3 Jan 2021

Copyright $\odot$ The authors 2021. This version is distributed under the terms of the Creative Commons Attribution NonCommercial Licence 4.0. For commercial reproduction rights and permissions contact permissions@ersnet.org 


\section{Introduction}

Precision medicine or, more broadly, precision health is the approach in which preventive measures and/ or health care interventions are personalised and tailored to each individual's characteristics. These traits are believed to reflect risks and/or response to interventions, and effectively harnessing this model has long been a goal of healthcare. Indeed, over recent years, development in pharmacogenomics has made such targeted medicine more available. However, evidence for the value of such approaches, particularly in the context of common environmental exposures [1], is required to support their implementation. Also, in the face of limited healthcare resources, the decision to allocate resources to an intervention is associated with "opportunity costs" of not being able to allocate resources to other ones. As such, in addition to clinical benefit, economic evaluation needs to be considered to determine when personalised medicine can be applied [2]. A comprehensive analysis demonstrating the "value for money" potential of using pharmacogenomics methods to guide health care interventions can support informed decision making in the adaptation of these technologies in clinical practice and prioritising research. This study proposes a modelling framework for predicting the costs and effectiveness of personalised health in the context of genotyping for the prevention of asthmatics exposed to air pollution.

Asthma is one of the most common chronic diseases worldwide, including in Canada and British Columbia, where it poses a significant economic burden $[3,4]$. Asthma is a multifactorial disease whose risk and burden is a complex function of both genetic and environmental factors. Multiple studies have shown that exposure to air pollution can impact lung function and worsen asthma symptoms [5-7].

In recent years, British Columbia has experienced longer and more severe fire seasons and more frequent forest fires, which severely impact air quality. The effect of forest fire-generated smoke exposures in British Columbia and its adverse effect on public health, including an increase in physician visits and hospital admissions for respiratory and cardiovascular diseases, has been demonstrated [8-12]. However, not all asthmatics have a similar risk of being affected by such exposures. For example, there is evidence showing that glutathione-S-transferases (GSTs) genes can modify the impact of air pollution on lung function as well as the risk and severity of asthma and allergy [7,13-16].

In this context, designing personalised preventive therapy for those exposed to air pollution, based on genotyping, can potentially improve quality of life and save costs. To inform such personalising of care, we conducted a cost-effectiveness analysis to predict the costs and benefits of proposed preventive strategies including personalised preventive intervention to reduce the adverse effect of known air pollution events such as a forest fire in asthmatics with mild disease. While the modelling framework developed and utilised in this study was based on data from British Columbia, it can be used in other settings to analyse cost-utility of personalised preventive intervention in settings of acute air pollution and, with model adaptation, other exposures.

\section{Methods}

In reporting the results of this study, we have adhered to Consolidated Health Economic Evaluation Reporting Standards (CHEERS) [17].

\section{Model overview}

A decision-analytic model was used to conduct a cost-utility analysis of prevention interventions in case of acute exposure to air pollution, such as wildfire smoke in seasonal forest fires, in mild asthma. The schematic diagram of the simulation model is illustrated in figure 1 . Key parameters of the model, including unit costs, utility weights, and main parameters for risk and prevalence of the asthma cohort are presented in table 1 .

We employed a probabilistic analysis to predict costs and QALYs associated with each decision, and incorporated uncertainty in the evidence base. The model was developed using $\mathrm{R}$ statistical software version 3.4 [26]. The analysis was from a Canadian public health care payer perspective with a one-year time horizon. Due to the one-year horizon, discounting was not applied to either costs or benefits.

The target population comprised patients with mild asthma which, according to the Global Initiative for Asthma (GINA) guidelines, is defined as asthma that is well controlled with as-needed reliever medication alone, or with low-intensity controller intervention such as low-dose inhaled corticosteroids (ICS), leukotriene receptor antagonists or cromolyn [27].

The model was developed based on the following assumptions: a) exposure to air pollution can lead to an asthma exacerbation (including those requiring an emergency room visit) in asthmatics with a genetic abnormality; b) prescribing additional medication as a prevention therapy decreases the risk of such exacerbation; c) asthmatics with mild asthma who are identified as normal genotype will carry no extra risk of exacerbation due to air pollution. 


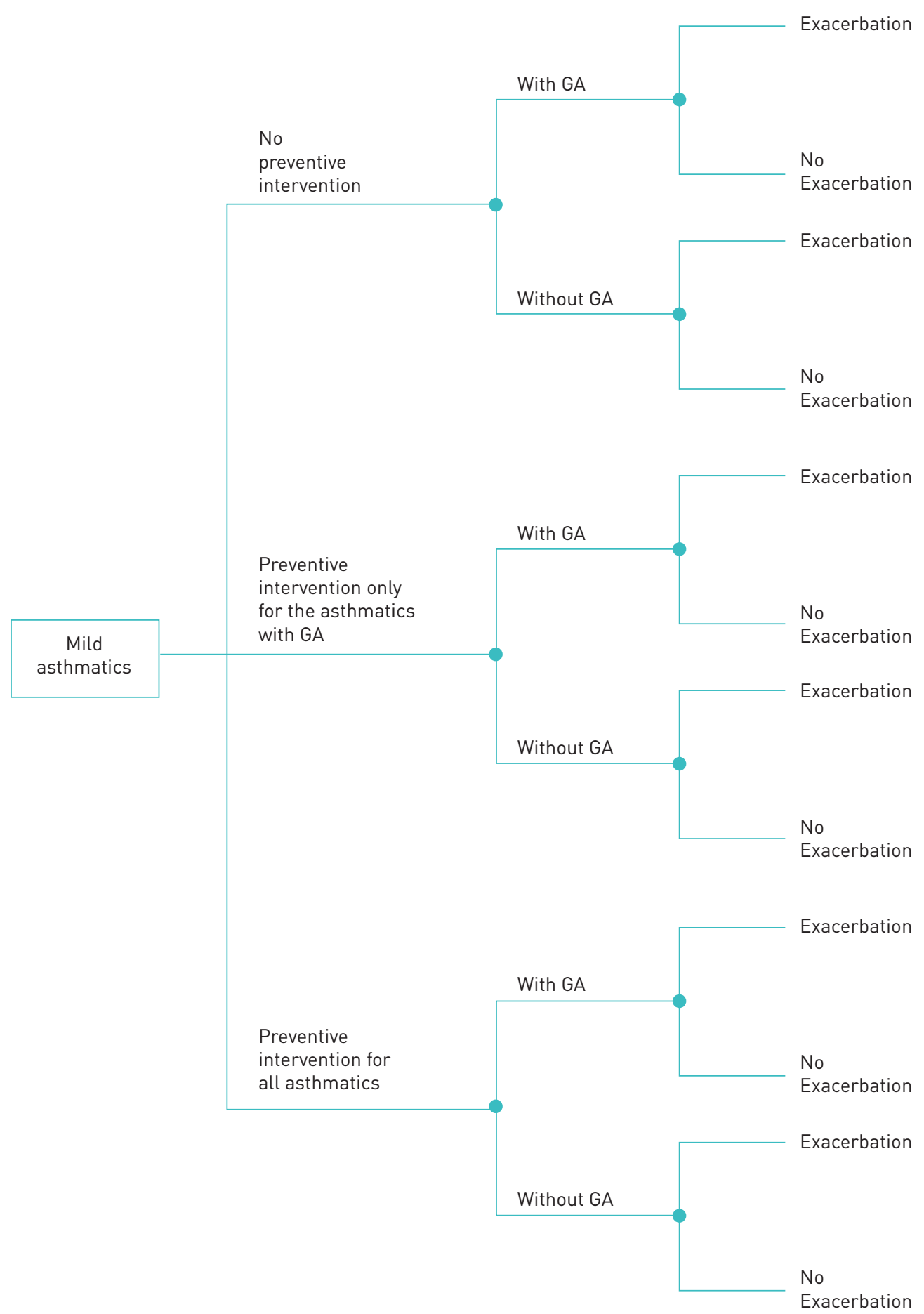

FIGURE 1 Schematic diagram of the model. GA: genetic abnormality.

The modelled intervention is prescribing additional ICS and long-acting $\beta$-agonists (LABA) (Advair 500/50 $\mu \mathrm{g}$ twice a day) for 2 months each summer. The intervention model begins at the start of the fire season (July 1 ). Genetic abnormality was defined as having one of the following genotypes: GSTT1 null or GSTM1 null or GSTP1 Ile105Val. These were chosen based on their well-established influence on clinically relevant respiratory responses to air pollution, including acute decrements in lung function and associated disease exacerbations $[6,7,14,28,29]$.

We modelled and compared three different strategies: the first strategy is no preventive intervention (standard of care); the second strategy is the precision health strategy based on information from genotype 


\begin{tabular}{|c|c|c|c|}
\hline Parameter & Mean & Distribution & Reference \\
\hline \multicolumn{4}{|l|}{ Probabilities } \\
\hline Prevalence of asthma & 323500 & Fixed & [19] \\
\hline Percentage of mild asthma & $67.1 \%$ & Fixed & [4] \\
\hline $\begin{array}{l}\text { Prevalence of genetic abnormality (GSTT1 null, GSTM1 } \\
\text { null or GSTP1 Ile105) }\end{array}$ & $25 \%$ & Fixed & {$[20,21]$} \\
\hline $\begin{array}{l}\text { Risk of additional exacerbation in asthmatics with } \\
\text { genetic abnormality, without preventive intervention }\end{array}$ & $55 \%$ & Beta $(42.7,34.9)$ & {$[22,23]$} \\
\hline $\begin{array}{l}\text { Risk of additional exacerbation in asthmatics with } \\
\text { genetic abnormality, with preventive intervention }\end{array}$ & $5 \%$ & Beta $(91.2,1732.5)$ & {$[22,23]$} \\
\hline $\begin{array}{l}\text { Risk of additional exacerbation in asthmatics without } \\
\text { genetic abnormality (with or without preventive } \\
\text { intervention) }\end{array}$ & 0 & Fixed & \\
\hline \multicolumn{4}{|l|}{ Unit costs } \\
\hline Advair 500/50 twice per day (per month) & $\$ 74.7$ & Fixed & [19] \\
\hline Exacerbation without hospitalisation & $\$ 126$ & Gamma $(96.04,0.76)$ & $\begin{array}{l}\text { [24] } \\
\text { (Converted to } \\
2018 \text { CAD) }\end{array}$ \\
\hline Exacerbation requiring emergency room visits & $\$ 575$ & Gamma $(96,0.17)$ & $\begin{array}{l}\text { [24] } \\
\text { (Converted to } \\
2018 \text { CAD) }\end{array}$ \\
\hline Exacerbation requiring hospitalisations & $\$ 6440$ & Gamma $(96,0.014)$ & $\begin{array}{l}\text { [24] } \\
\text { (Converted to } \\
2018 \text { CAD) }\end{array}$ \\
\hline Genetic testing & 109.43 & Fixed & $\begin{array}{l}\text { [25] } \\
\text { (Converted to } \\
2018 \text { CAD) }\end{array}$ \\
\hline \multicolumn{4}{|l|}{ Utilities } \\
\hline Exacerbation without hospitalisation & 0.57 & Beta $(0.51,0.38)$ & {$[26]$} \\
\hline Exacerbation requiring emergency room visits & 0.45 & Beta $(0.36,0.45)$ & {$[26]$} \\
\hline Exacerbation requiring hospitalisations & 0.33 & Beta $(0.15,0.30)$ & {$[26]$} \\
\hline
\end{tabular}

testing. In this strategy, the preventive intervention (Advair 500/50 twice a day) is prescribed only to asthmatics with mild asthma who are identified as having a genetic abnormality in the genotype test; and the third strategy includes prescribing additional medication (Advair 500/50 twice a day) to all mild asthmatics as a preventive intervention.

\section{Unit costs}

The costs of an asthma exacerbation were derived from a previous study [23]. We used a weighted average of the cost of exacerbation without hospitalisation and exacerbation requiring emergency room visits. The cost of genotyping was based on published data [24]. Canadian costs were used directly where available. Otherwise, available US costs were converted to Canadian costs using a two-step approach: first, we applied the ratio of healthcare expenditure in the USA and Canada [30]; second, the consumer price index for healthcare was used to adjust costs to 2018 Canadian dollars (CAD).

\section{Utility weights}

Utility weights for different levels of exacerbation severity came from a study conducted in the UK [25]. A weighted average of utility weights for exacerbation without hospitalisation and exacerbation requiring emergency room visits was used in the model. Effectiveness was measured in terms of change in QALY loss.

\section{Sensitivity and uncertainty analysis}

In addition to the base case analysis, we conducted scenario analyses considering different modelling assumptions and investigated the effects of these assumptions. To address uncertainty around the value of input parameters included in the model and examine the impact of this uncertainty on the outcomes, we adopted a probabilistic approach in the base case and scenario analyses. In probabilistic analysis, input parameters are represented as distributions that reflect the level of uncertainty (e.g. constructed form the point estimate and confidence intervals from original studies). Monte Carlo simulation was then applied to 
propagate the uncertainty through the model. This technique uses random numbers to sample from the input data probability distributions. As such, this method of analysis allows quantifying the level of confidence in the output of the model. Following standard practice, gamma distributions were assigned to the costs, and the probabilities were drawn from beta distributions [31].

\section{Analysis}

The expected values of costs and QALYs in the base case and all scenario analyses were obtained through probabilistic analysis from the Monte Carlo simulation. We ran the analysis 10000 times, and each time different random values were selected for the inputs of the model, resulting in 10000 sets of results from which the statistical output data are derived. Finally, we extrapolated these results to the entire population of British Columbia to estimate the number of exacerbations that can be avoided using a preventive intervention was derived.

\section{Results}

Base case analysis

The results of a sequential cost-utility analysis of comparing three strategies, including 1) No intervention, 2) preventive intervention only for asthmatics with genetic abnormality, and 3) preventive intervention for all asthmatics, are presented in table 2 .

\section{No intervention compared to preventive intervention only for asthmatics with genetic abnormality}

Comparing no intervention with targeted therapy shows that using a preventive intervention only for the ones with genetic abnormality is expected to cost more than no intervention ( $\$ 150.5$ versus $\$ 42)$ but it causes QALY gain (decrease in QALY loss from 0.0024 to 0.00021). The incremental cost-effectiveness ratio (ICER) was $\$ 49555$ per QALY. At a willingness-to-pay threshold of $\$ 50000$ per QALY, the personalised intervention is considered cost-effective (table 2).

Results of comparing no intervention and treating only the asthmatics with genetic abnormality are also presented in the cost-effectiveness acceptability curve (CEAC). CEAC shows the probability of the targeted intervention being cost-effective compared with no intervention across a range of willingness-to-pay thresholds.

As shown in CEAC (figure 2) there is a 50\% probability that the ICER would be less than $\$ 50000$ per QALY and a 75\% probability of the ICER being less than $\$ 100000$ per QALY.

\section{Preventive intervention for asthmatics with genetic abnormality compared with preventive intervention for all asthmatics}

As shown in table 2, preventive intervention only for asthmatics with genetic abnormality compared with preventive intervention for all asthmatics lowered cost for healthcare system ( $\$ 150$ versus $\$ 153$ ) while the change in QALY was the same. As such, preventive intervention only for the ones with GA is a dominant strategy.

\section{Population-level outcome}

Considering the estimate of mild asthmatics in British Columbia and the average prevalence of genetic abnormality (table 1), we project that 27093 extra pollution-induced exacerbations can be avoided in 1 year by using additional medication as a preventive intervention.

\section{Scenario analyses}

We also conducted a series of scenario and sensitivity analyses to explore the impact of uncertainty around the value of the model's parameters and alternative assumptions on the outcomes of the model. To

TABLE 2 The expected value of costs, QALY and incremental cost per QALY (base case) per one patient with mild asthma

\begin{tabular}{|c|c|c|c|c|c|}
\hline Strategy & Costs $(\$)$ & $\begin{array}{l}\text { QALY } \\
\text { loss }\end{array}$ & $\begin{array}{c}\text { Sequential } \\
\text { incremental costs }\end{array}$ & $\begin{array}{c}\text { Sequential incremental } \\
\text { QALY loss }\end{array}$ & $\begin{array}{l}\text { Sequential incremental } \\
\text { cost per QALY Gained }\end{array}$ \\
\hline No intervention & 42.13 & 0.0024 & & & \\
\hline Preventive intervention for all asthmatics & 153.2 & 0.00021 & 2.61 & 0 & Dominated \\
\hline
\end{tabular}




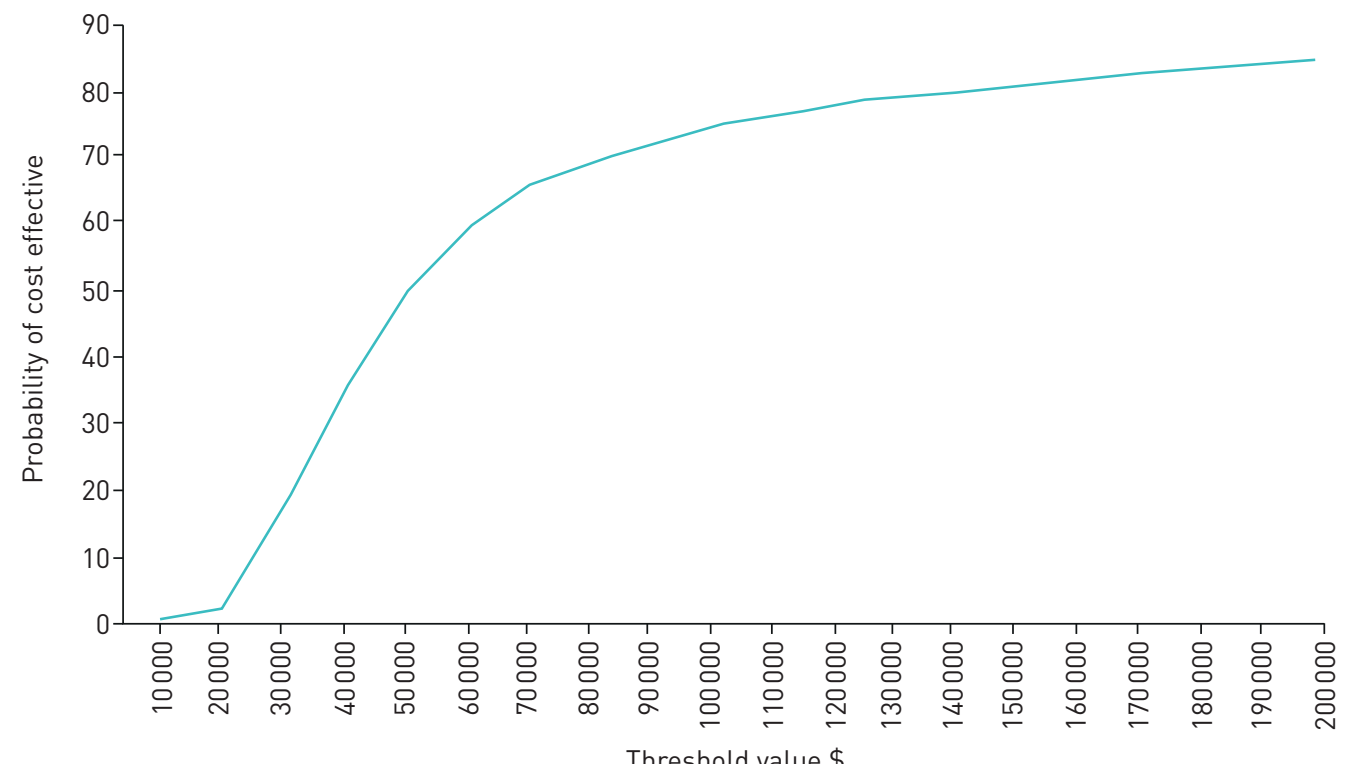

FIGURE 2 Cost-effectiveness acceptability curve comparing no intervention with targeted intervention.

simplify reporting, for the scenario/sensitivity analyses, we compared the preventive intervention (intervention only for the asthmatics with genetic abnormality) with no intervention. The results are presented in Supplementary Table 1 and figure 3. The Tornado diagram in figure 3 illustrates graphically how variations in each input or assumption affect the outcome.

The sensitivity analysis that varied the prevalence of the genetic abnormality showed that results are sensitive to this parameter. When the prevalence of genetic abnormality was increased to $45 \%$, costs and QALY loss of both strategies went higher compared to the base case but ICER decreased to $\$ 27330$ per QALY, which again indicates that preventive intervention for asthmatics with genetic abnormality is cost effective. However, with the lower prevalence of genetic abnormality at $15 \%$, costs and QALY loss were lower for both strategies, but the ratio of cost and effectiveness changed to \$82894 per QALY and at a willingness-to-pay threshold of $\$ 50000$ per QALY, it is not cost-effective to prescribe intervention for the asthmatics with genetic abnormality compared to no intervention.

Results of analysis are also sensitive to the estimated risk of pollution-induced exacerbation in asthmatics with genetic abnormality (without preventive intervention). We assumed this risk may vary based on some factors like level of exposure to air pollution. When the risk was increased to 65\%, costs and QALY loss of

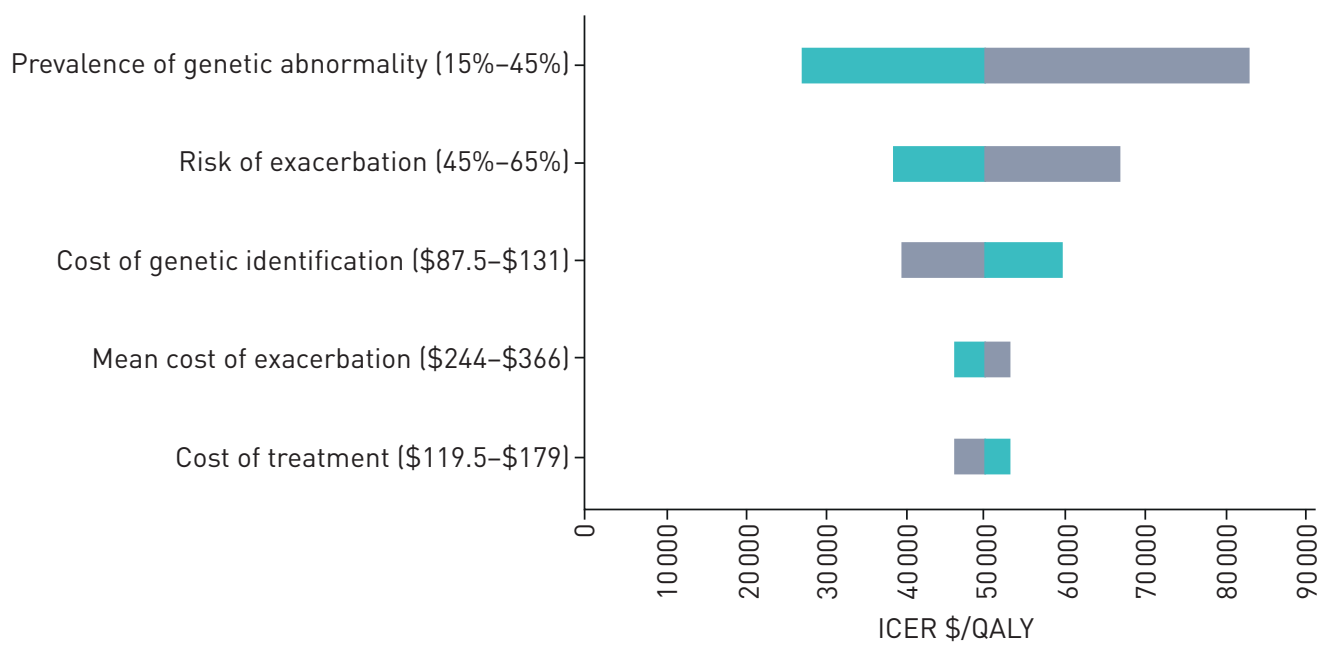

FIGURE 3 Scenario analysis (tornado diagram). ICER: incremental cost-effectiveness ratio; QALY: quality-adjusted life-year. 
no intervention strategy went higher compared to the base case, such that the suggested intervention remained cost effective (ICER of 38528 per QALY). On the other hand, when the risk of exacerbation was assumed lower than the base case (45\%), ICER was higher than the threshold (\$66844 per QALY) and the suggested intervention was no longer cost effective.

The cost of genetic identification also affects the results. When the cost of genetic testing is lower than the base case value, expected cost of the suggested strategy is decreased, which results in a lower ICER, thus the suggested intervention remains cost-effective. With the assumed cost of $\$ 0$ for the cost of genetic testing, the suggested intervention becomes a dominant strategy compared to no intervention as the cost is lower and efficiency is increased (Supplementary Table 1). Similarly, the higher cost of genetic identification will increase ICER. Next, as is demonstrated in Supplementary Table 1, when the mean cost of exacerbation was lower compared to the base case, the ICER was higher and with the higher mean cost of exacerbation, and the ICER was lower compared to the base case which means the suggested intervention remains cost-effective (Supplementary Table 1).

\section{Discussion}

To our knowledge, this study is the first to perform an economic evaluation of a specific application of precision health to preventing exacerbations of asthma. Importantly, we framed the analysis around a common scenario (exposure to air pollution in asthmatics) with broad public health relevance. The decision-analysis model employed in the current study provides a framework for the cost-effectiveness of preventing pollution-induced asthma exacerbations based on genotype testing. The model assessed the incremental cost-effectiveness ratio of preventive intervention strategies and compared these with the current standard of care (no personalised preventive intervention). The findings show that the suggested intervention (anticipatory intervention for the asthmatics with relevant genetic abnormality facing acute air pollution) is a cost-effective strategy compared with no such intervention. Furthermore, this strategy is a dominant strategy compared with an intervention that prescribes medication indiscriminately (without regard to genotype) to all asthmatics. A series of scenario analyses showed that these outcomes are most sensitive to the prevalence of genetic abnormality among asthmatics, risk of exacerbation in asthmatics with genetic abnormality, and to the cost of genetic testing.

Our study has some limitations, which generally lead to a tendency to underestimate the benefits of a precision approach to prevention in our example. First, in the model, we considered the risk of one additional exacerbation in the context of increased air pollution exposure whereas, in reality, pollution-related exacerbations can happen more than once in a year. Since effectiveness of the preventive intervention will increase along with the risk of multiple exacerbations, our method has therefore generated conservative results that may underestimate the benefits. Second, there is uncertainty around some of the key parameters of the model, such as the prevalence of genetic abnormality in the exposure population. In order to overcome this issue, we used probabilistic analyses and a series of scenario analyses to support the generalisability of our results. Even so, this approach still likely leads further to an underestimation of the benefit of preventive pharmacotherapy, because the true population includes those with more than one of the genetic abnormalities assessed. Multiple such abnormalities lead to increased susceptibility to pollution [6] and, accordingly, likely addition benefits from the preventive intervention, but we did not have sufficient data on population prevalence therein for modelling. Third, in the model, we have assumed that our target group of mild asthmatics don't develop an exacerbation requiring hospitalisation; although exacerbations even in mild asthmatics can sometimes lead to hospitalisation, we have again taken a conservative approach based on the likelihood that if a mild asthmatic were to develop an exacerbation requiring hospitalisation, he/she would no longer fit the definition of mild asthma. Furthermore, as genotyping is inherently stable and so needs be done once, for subsequent years the ICER will be even more favourable. Finally, we have used July 1 as the start of the fire season, whereas it often starts considerably earlier (in which case the benefit of precision intervention would be enhanced). Collectively, this suggests that the precision health approach modelled may confer considerable cost-effectiveness benefit even beyond that shown in our primary analysis. That said, our analysis represents a proof-of-principle that is not intended for direct decision-making without further work to refine and update the parameters inputted into the model.

\section{Conclusion}

Our findings provide evidence that the incorporation of genomic testing, to stratify risk level of asthmatics to pollution-driven exacerbations, and tailoring a preventive intervention accordingly, may be cost-effective relative to usual care. The results inform and motivate the design of programmes that use a precision health framework to limit asthma exacerbation in the context of acute air pollution excursions and, furthermore, serve as a model of other genotype-driven preventive strategies for a range of common exposures. 
Acknowledgements: C. Carlsten is supported by the Canada Research Chairs programme. In reporting the results of this study, we have adhered to Consolidated Health Economic Evaluation Reporting Standards (CHEERS).

Author contributions: All authors provided equal contributions to the preparation of this manuscript.

Conflict of interest: None declared.

Support statement: Support for this project came from AllerGen NCE (project GxE4) and the Legacy for Airway Health (https://www.vchri.ca/legacyforairwayhealth). Funding information for this article has been deposited with the Crossref Funder Registry.

\section{References}

1 Carlsten C, Brauer M, Brinkman F, et al. Genes, the environment and personalized medicine: We need to harness both environmental and genetic data to maximize personal and population health. EMBO Rep 2014; 15: 736-739.

2 Hatz MHM, Schremser K, Rogowski WH. Is individualized medicine more cost-effective? A systematic review. PharmacoEconomics 2014; 32: 443-455.

3 Ismaila AS, Sayani AP, Marin M, et al. Clinical, economic, and humanistic burden of asthma in Canada: a systematic review. BMC Pulm Med 2013; 13: 70.

4 Sadatsafavi M, Lynd L, Marra C, et al. Direct health care costs associated with asthma in British Columbia. Can Respir J 2010; 17: 74-80.

5 Namork E, Johansen BV, Løvik M. Detection of allergens adsorbed to ambient air particles collected in four European cities. Toxicol Lett 2006; 165: 71-78.

6 Wooding DJ, Ryu MH, Hüls A, et al. Particle depletion does not remediate acute effects of traffic-related air pollution and allergen. A randomized, double-blind crossover study. Am J Respir Crit Care Med 2019; 200: 565-574.

7 Zhang X, Hirota JA, Yang C, et al. Effect of GST variants on lung function following diesel exhaust and allergen co-exposure in a controlled human crossover study. Free Radic Biol Med 2016; 96: 385-391.

8 Elliott CT, Henderson SB, Wan V. Time series analysis of fine particulate matter and asthma reliever dispensations in populations affected by forest fires. Environ Health 2013; 12: 11.

9 Henderson SB, Brauer M, Macnab YC, et al. Three measures of forest fire smoke exposure and their associations with respiratory and cardiovascular health outcomes in a population-based cohort. Environ Health Perspect 2011, 119: $1266-1271$.

10 McLean KE, Yao J, Henderson SB. An evaluation of the British Columbia Asthma Monitoring System (BCAMS) and PM2.5 exposure metrics during the 2014 forest fire season. Int J Environ Res Public Health 2015; 12: $6710-6724$.

11 Moore D, Copes R, Fisk R, et al. Population health effects of air quality changes due to forest fires in British Columbia in 2003: estimates from physician-visit billing data. Can J Public Health 2006; 97: 105-108.

12 Yao J, Brauer M, Henderson SB. Evaluation of a wildfire smoke forecasting system as a tool for public health protection. Environ Health Perspect 2013; 121: 1142-1147.

13 Gilliland FD, Li Y-F, Saxon A, et al. Effect of glutathione-S-transferase M1 and P1 genotypes on xenobiotic enhancement of allergic responses: randomised, placebo-controlled crossover study. Lancet 2004; 363: 119-125.

14 MacIntyre EA, Brauer M, Melén E, et al. GSTP1 and TNF gene variants and associations between air pollution and incident childhood asthma: the Traffic, Asthma and Genetics (TAG) study. Environ Health Perspect 2014; 122 $418-424$

15 North ML, Alexis NE, Ellis AK, et al. Air pollution and asthma: how can a public health concern inform the care of individual patients? Ann Allergy Asthma Immunol 2014; 113: 343-346.

16 Ren C, Park SK, Vokonas PS, et al. Air pollution and homocysteine: more evidence that oxidative stress-related genes modify effects of particulate air pollution. Epidemiol Camb Mass 2010; 21: 198-206.

17 Husereau D, Drummond M, Petrou S, et al. Consolidated Health Economic Evaluation Reporting Standards (CHEERS)-explanation and elaboration: a report of the ISPOR Health Economic Evaluation Publication Guidelines Good Reporting Practices Task Force. Value Health 2013; 16: 231-250.

18 Health M of. Asthma in Adults - Recognition, Diagnosis and Management - Province of British Columbia Province of British Columbia. www2.gov.bc.ca/gov/content/health/practitioner-professional-resources/bcguidelines/asthma-adults?keyword=asthma Date last accessed: June 16, 2020; date last updated: October, 2015.

19 Minelli C, Granell R, Newson R, et al. Glutathione-S-transferase genes and asthma phenotypes: a Human Genome Epidemiology (HuGE) systematic review and meta-analysis including unpublished data. Int J Epidemiol 2010; 39: 539-562.

20 Tamer L, Çalikoğlu M, Ates NA, et al. Glutathione-S-transferase gene polymorphisms (GSTT1, GSTM1, GSTP1) as increased risk factors for asthma. Respirology 2004; 9: 493-498.

21 Nelson HS. Advair: Combination treatment with fluticasone propionate/salmeterol in the treatment of asthma. J Allergy Clin Immunol 2001; 107: 397-416.

22 Orellano P, Quaranta N, Reynoso J, et al. Effect of outdoor air pollution on asthma exacerbations in children and adults: systematic review and multilevel meta-analysis. PLoS One 2017; 12: e0174050.

23 Zafari Z, Sadatsafavi M, Marra CA, et al. Cost-effectiveness of bronchial thermoplasty, omalizumab, and standard therapy for moderate-to-severe allergic asthma. PLoS One 2016; 11: e0146003.

24 Bielinski SJ, St. Sauver JL, Olson JE, et al. Are patients willing to incur out of pocket costs for pharmacogenomic testing? Pharmacogenomics J 2017; 17: 1-3.

25 Campbell JD, Spackman DE, Sullivan SD. The costs and consequences of omalizumab in uncontrolled asthma from a USA payer perspective. Allergy 2010; 65: 1141-1148.

26 R Core Team. R: A language and environment for statistical computing. Vienna, Austria, R Foundation for Statistical Computing, 2018. www.R-project.org.

27 Global Initiative for Asthma. 2019 GINA Main Report. https://ginasthma.org/gina-reports/ Dast last accessed: December 11, 2020; date last updated: 2020. 
28 Carlsten C, Blomberg A, Pui M, et al. Diesel exhaust augments allergen-induced lower airway inflammation in allergic individuals: a controlled human exposure study. Thorax 2016; 71: 35-44.

29 Sava F, Carlsten C. Respiratory health effects of ambient air pollution: an update. Clin Chest Med 2012; 33: 759-769.

30 Lorenzoni L, Belloni A, Sassi F. Health-care expenditure and health policy in the USA versus other high-spending OECD countries. Lancet 2014; 384: 83-92.

31 Briggs A, Sculpher M, Claxton K. Decision Modelling for Health Economic Evaluation. Oxford, Oxford University Press, 2006. 